\title{
Expression analysis of mesenchymal KS483 cells during differentiation towards osteoblasts
}

\author{
Igor Fijalkowski1, Vere Borra ${ }^{1}$, Eveline Boudin ${ }^{1}$, Wim Van Hul${ }^{1}$
}

${ }^{1}$ Department of Medical Genetics, University of Antwerp, Belgium

\section{Introduction}

The Murine osteoprogenitor cell line, KS483 (Percuros, The Netherlands) is a well-established model for investigation of osteoblast differentiation and bone formation processes. The mesenchymal characteristics of this cell line allow it to differentiate into either adipocytes or mature, mineralizing osteoblasts. Various phases can be distinguished during osteoblast differentiation and maturation; namely proliferation, matrix formation, matrix maturation, and mineralization.

We now performed whole genome expression analysis of mRNA in order to gain additional insight into the molecular mechanisms driving these processes with the focus on Wnt/ $\beta$-catenin signaling involvement. We investigate the expression patterns of core elements of this pathway, together with its known regulators.

We hope that with this study we will be able to develop a reference dataset for osteoblast differentiation experiments and gain insight into the biology of undelying processes.

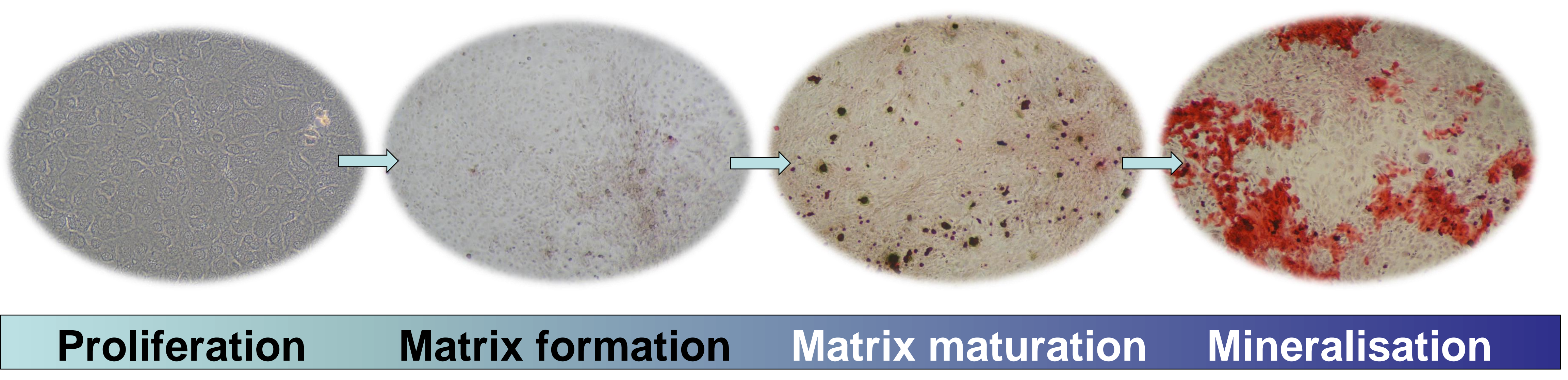

\section{Results}

Expression data:

- On average 7400 genes displayed robust expression at each time point (detection p-value $<0.01$ )

- In comparison to day 4 (stationary state) 2539 genes display statistically significant changes in expression at day 28 .

Differentiation process:

- Bone formation markers follow logical expression pattern

- Despite being differentiated for 28 days, the cells remained negative for osteocyte markers: Mepe, Sost, Dmp1, Phex

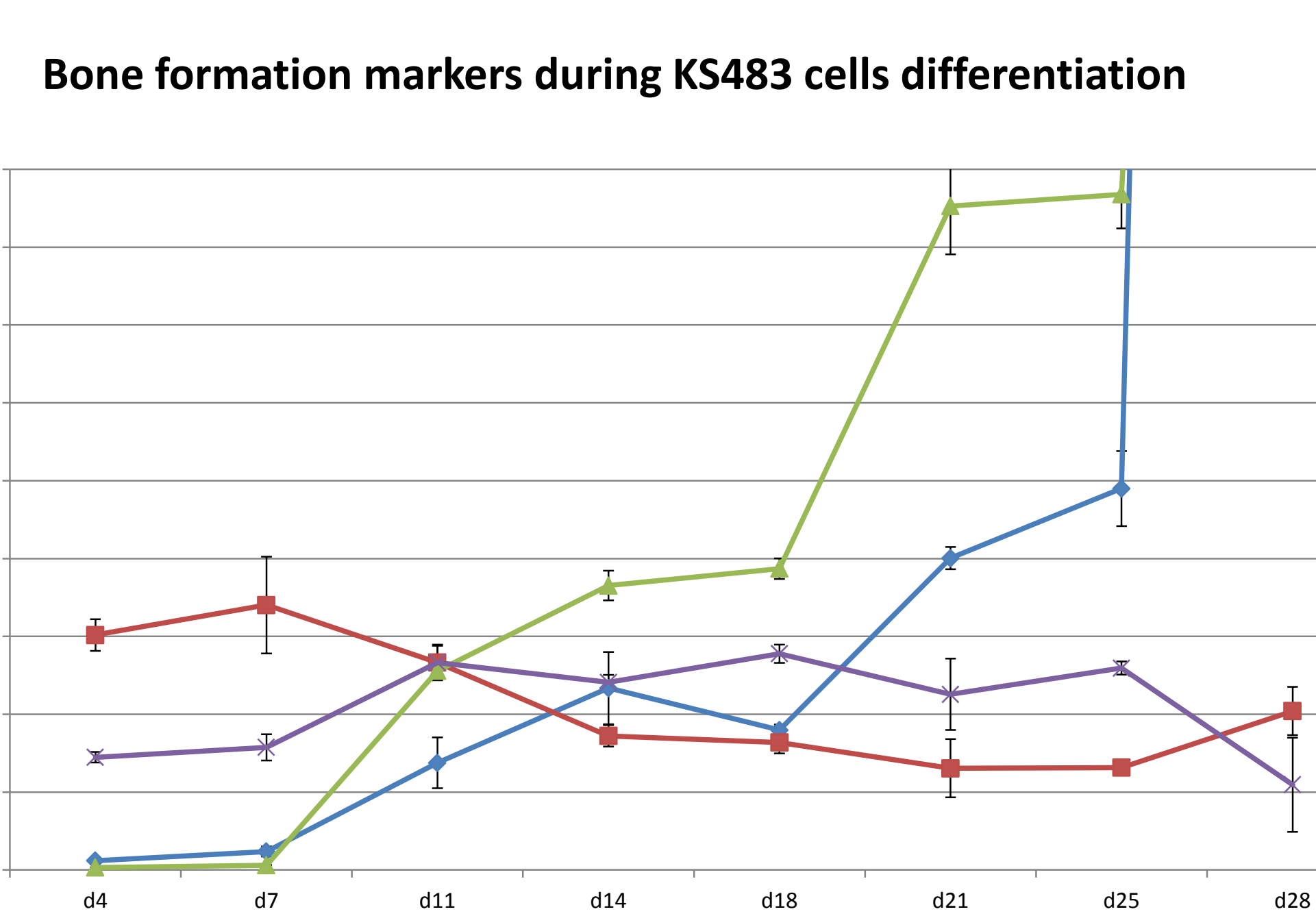

Wnt genes:
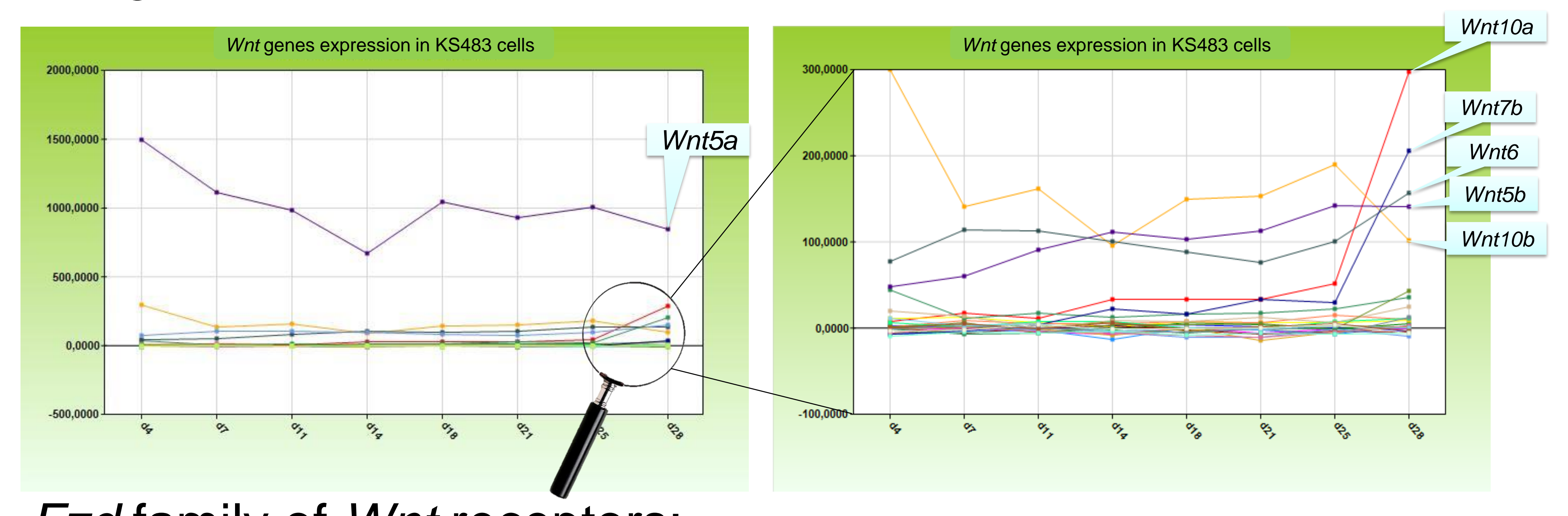

Fzd family of Wnt receptors:
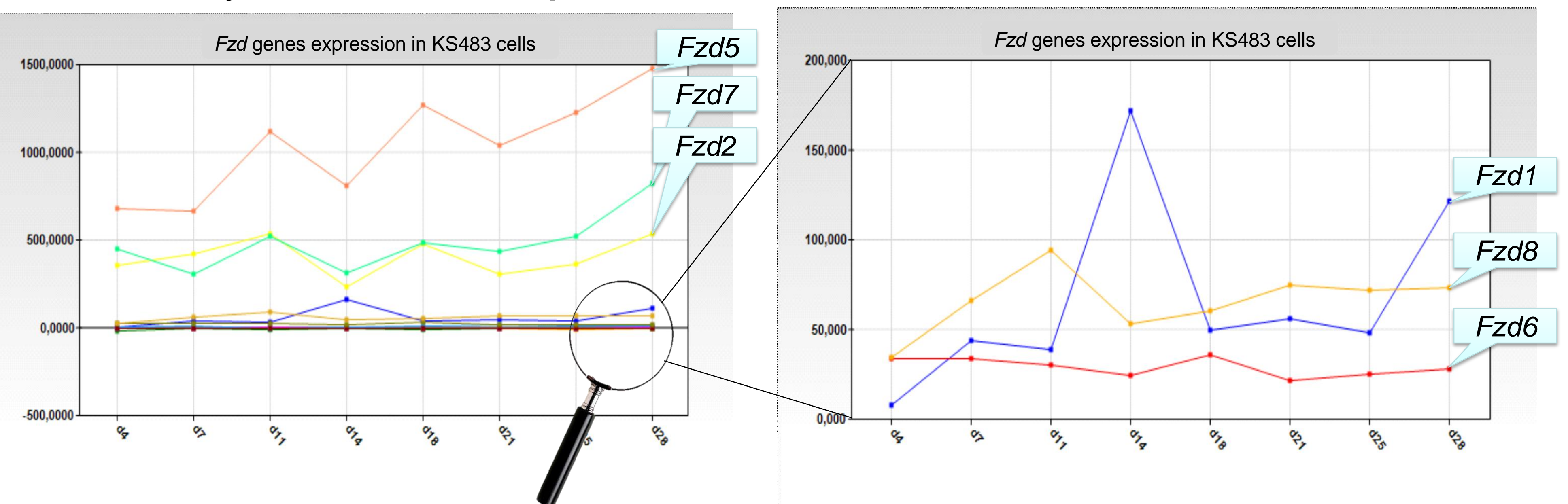

Lrp family of Wnt co-receptors
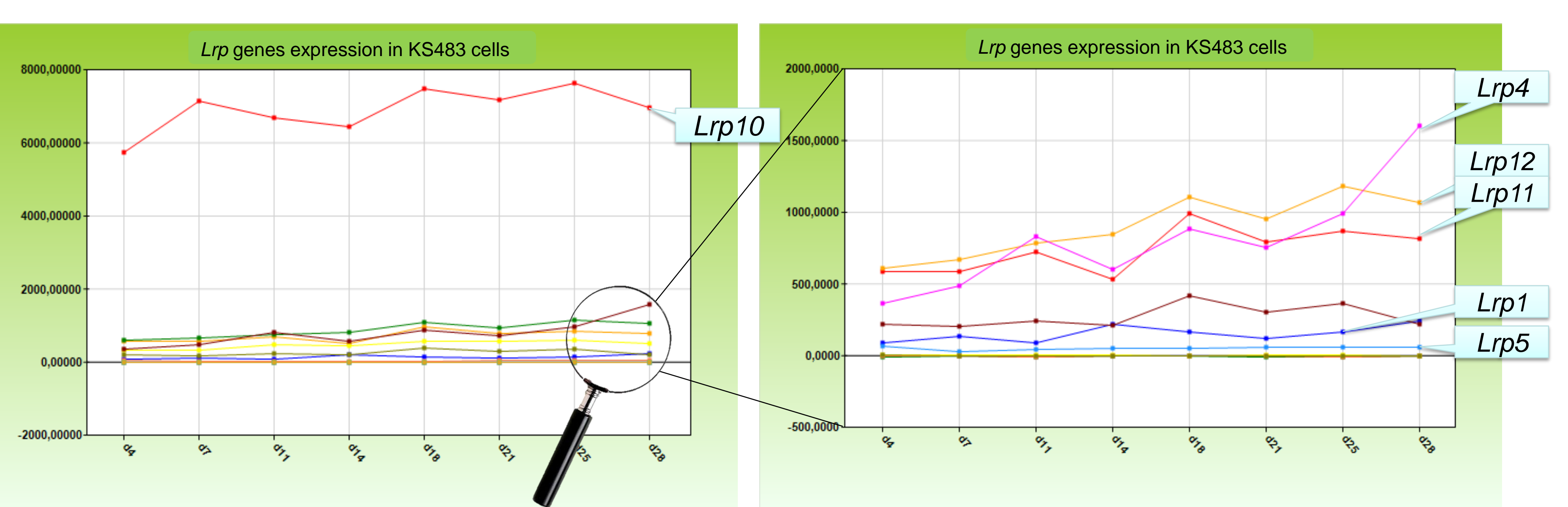

\section{Materials and methods}

KS483 cells were differentiated for 28 days in two biological replicates. Culture medium was supplemented with ascorbic acid (at day 4) and $\beta$-glycerolphosphate (day 11 onward). Total cellular RNA was isolated at 8 time points during this process and cRNA was generated with the use of Illumina TotalPrep RNA amplification Kit.

Expression of over 19100 unique genes was assessed with the use of MouseRef-8 v2.0 Expression BeadChips and Illumina iScan system. Data were analysed with the use of Illumina GenomeStudio and beadarray $\mathrm{R}$ package.

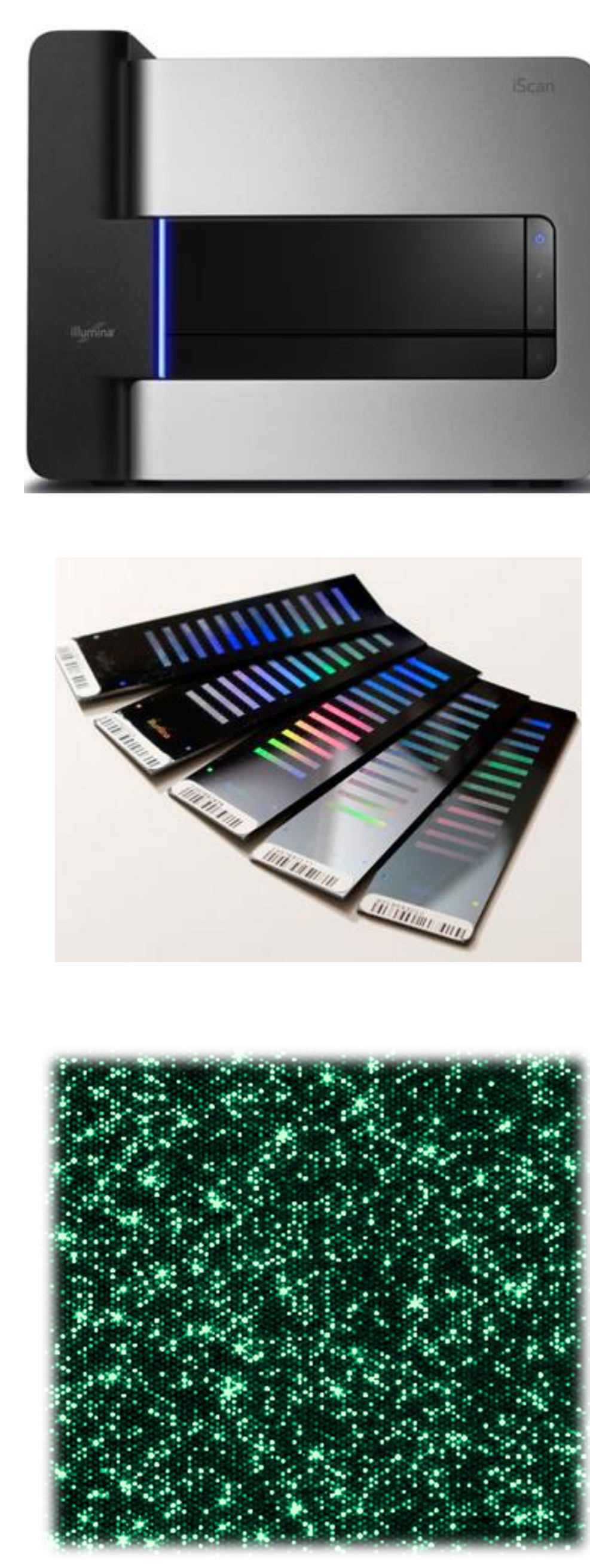

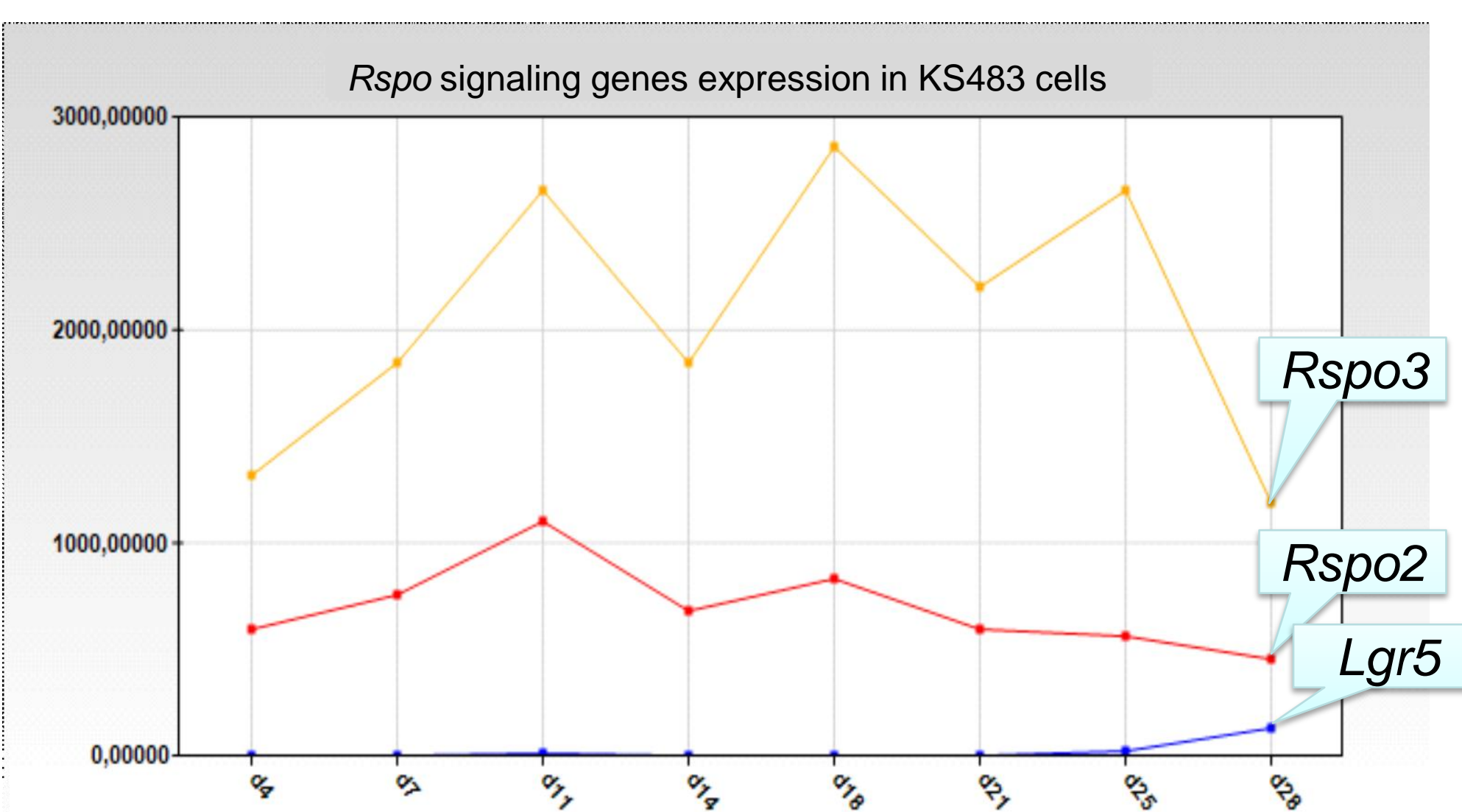
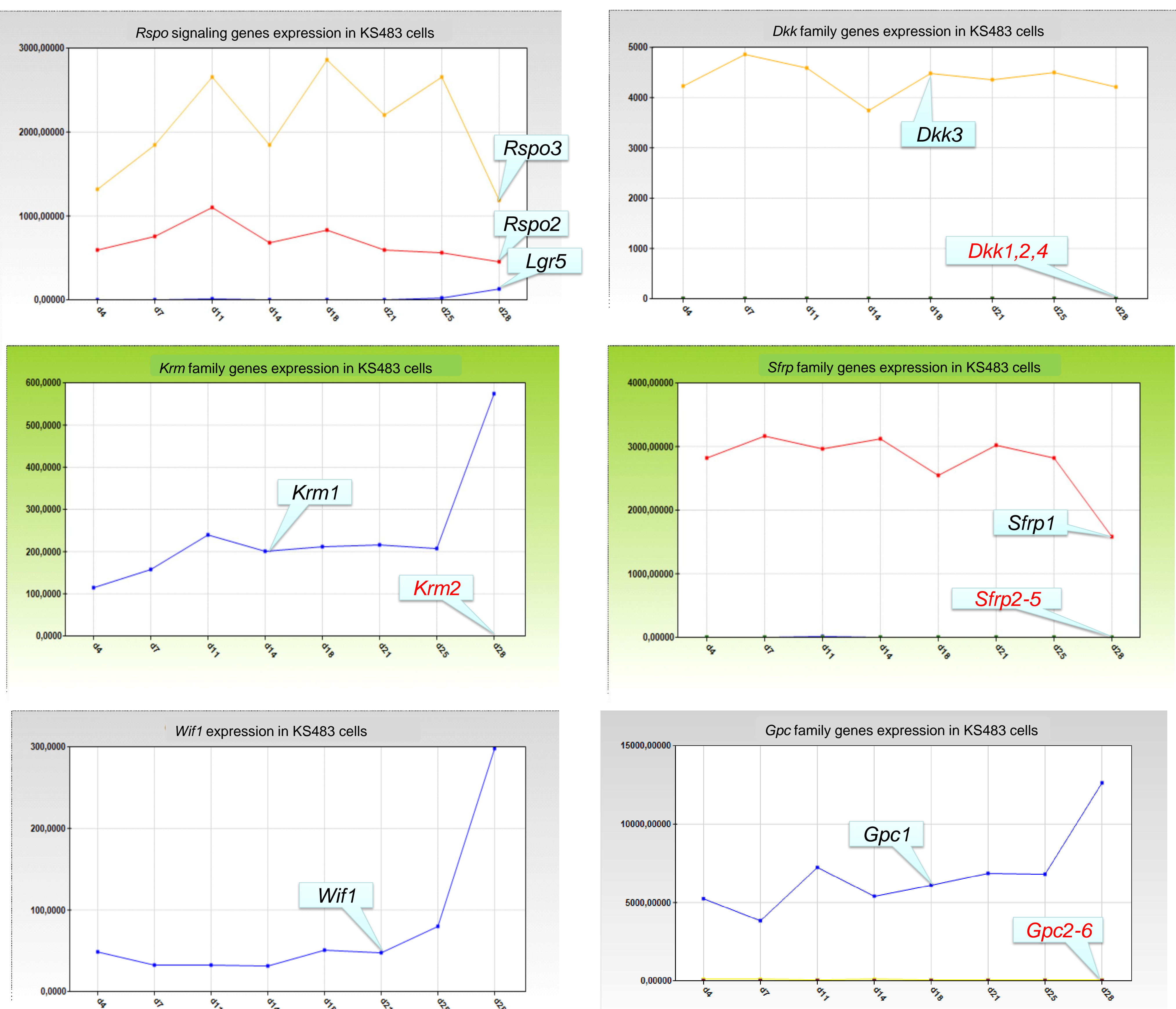

\section{Discussion \& Conclusion}

We believe that we were able to provide a useful and informative tool for the investigation of osteoblast differentiation and bone formation. We believe that it grants valuable insight into the molecular mechanisms underlying these processes. We anticipate that it will prove its utility in overexpression and silencing studies in KS483 cells, serving as the expression data reference and allowing for more detailed description of the transgene effect on the osteoblasts biology. 